\title{
液压系统流体脉动主动控制方法研究现状
}

\author{
商 夏 周 华 杨华勇 \\ (浙江大学流体动力与机电系统国家重点实验室 杭州 310027)
}

\begin{abstract}
摘要: 液压系统的流量脉动和压力脉动始终以耦合关系存在于系统中, 导致系统的控制精度下降和元件疲劳损失加快等问题。 由于液压系统具有参数时变和负载多变等特点, 相比于流体脉动的被动控制方法, 主动控制能更好地满足复杂非线性系统对 动态控制精度的要求。根据次级脉动源的不同, 分析主动控制的工作原理和控制特点, 列举四种在液压系统流体脉动主动控 制中常用的控制算法及其应用场合。此外，以伺服作动筒控制、液压阀控制和非侵入式结构控制进行分类，分析国内外液压 系统流体脉动主动控制方法的研究成果。总结流体脉动主动控制方法的研究现状与未来发展方向。
\end{abstract}

关键词: 主动控制; 流体脉动; 液压系统; 消振; 自适应控制

中图分类号: TH137

\section{Research Status of Active Control of Hydraulic Fluid Pulsation}

\author{
SHANG Xia ZHOU Hua YANG Huayong
}

(State Key Laboratory of Fluid Power and Mechatronic Systems, Zhejiang University, Hangzhou 310027)

\begin{abstract}
The flow pulsation and pressure pulsation always exist in hydraulic systems in a coupling relationship, which leads to the problems of the control accuracy decrease of the system and the accelerated fatigue loss of the components. Due to the characteristics of time-varying parameters and variable load of the hydraulic system, active control can better meet the requirements of the dynamic control accuracy of the complex nonlinear system compared to passive control for fluid pulsation. Based on different secondary pulsating sources, the working principle and the control characteristics of the active control methods are analyzed, and four common control algorithms and their application occasions for fluid pulsation active control in hydraulic systems are listed. In addition, the research results of the active control methods for fluid pulsation in hydraulic systems at home and abroad are analyzed by servo actuator control, hydraulic valve control and non-intrusive structural control. In the end, the research status and the future development directions of fluid pulsation active control are summarized.
\end{abstract}

Key words: active control; fluid pulsation; hydraulic systems; vibration attenuation; adaptive control

\section{0 前言}

基于惠更斯原理的主动振动控制和主动噪声控 制在诸多方面有相似之处 ${ }^{[1-2]}$ 。主动振动控制的最初 想法来源于德国物理学家 PAUL 在 20 世纪 30 年代 提出的主动噪声控制理论, 即利用传感器对系统产 生次级干扰以抵消初级噪声 ${ }^{[3]}$ 。此后, 主动振动控 制的研究被迅速展开, 在航空航天、土木工程、交 通运输工程等领域已被应用得颇为广泛且成熟, 例 如机翼颤振的稳定性控制、房屋的抗震设计、汽车 悬架的主动控制等。在液压系统中, 原的容积式结

* 国家自然科学基金创新研究群体(51521064)和浙江省重点科技创新团队 自主设计(2013TD01)资助项目。20180719 收到初稿, 20190316 收到修 改稿
构使液压系统管路内始终伴有流量脉动和压力脉 动, 耦合脉动容易引起系统元件和管路发生共振, 进而引发更大程度的振动和噪声。液压系统的流体 脉动控制对维持系统的稳定性、可靠性和控制精度 起到十分重要的作用。目前被动消振方法已较为成 熟, 例如加装赫姆霍兹共振器、蓄能器等, 但对于 主动消振方法的研究和应用正在进一步探索中, 具 有非常广阔的发展空间。

按是否需要能源, 振动控制可以分为无源(被动) 控制和有源(主动)控制 ${ }^{[4]}$ 。在液压系统中, 被动控制 的原理是通过对液压系统加装阻性衰减器、抗性衰 减器或阻抗复合式衰减器来改变系统阻抗, 从而达 到衰减系统流体脉动的目的 ${ }^{[5]}$ 。传统的消振方式多 为被动消振, 源于其无源廉价、简单易行、稳定可 靠且效果良好等优点 ${ }^{[6]}$ 。虽然被动消振能起到一定 
的脉动衰减作用, 但尚存在不足之处: (1) 液压系统 一般比较复杂且含有非线性元件、分布参数、时变 环节、不确定性等因素, 因此很容易受到外界干 扰的影响 ${ }^{[7]}$ 。被动消振无自适应性, 不能根据流 体脉动状态实时地调整控制参数, 因此通用性较 低; (2) 被动衰减器的结构参数相对固定, 即使可以 调整, 也只能是通过人工手调 ${ }^{[7]}$, 而且这种方法需 要在系统中额外增加开关阀等液压元件以防止油液 泄漏等问题; (3) 被动衰减器的体积通常较大, 安装 布局容易受到空间的限制, 同时也可能影响系统的 管路布局; (4) 被动衰减器的衰减频带窄, 通常对中、 高频段脉动的衰减效果较好, 对低频段脉动的衰减 效果不佳 ${ }^{[8]}$; (5) 基于扩张腔、蓄能器或软管等方式 的被动消振装置会造成静态压力损失, 从而影响系 统的动态特性和刚度 ${ }^{[9]}$ 。由此可见, 被动衰减器和 液压系统会形成相互制约的关系, 而且被动消振并 不能完全满足液压系统的实际工况需求。因此, 对 于工况多变且性能要求高的场合, 具有参数自适应 性、实时性以及衰减频带宽等优点的主动消振成为 目前液压系统脉动控制领域的研究热点。

本文首先阐述了液压系统流体脉动的产生机理 及危害, 进而对主动消振的基本原理、系统组成、 控制方法和控制算法进行了介绍。通过对各控制方 法的比对, 分别总结了其优点和不足, 并依据主动 消振方法分类列举了液压系统脉动控制领域的主要 研究成果。最后, 文章探讨了液压系统流体脉动主 动消振方法的未来发展方向。

\section{1 液压系统流体脉动产生机理及危害}

除利用振动原理工作的设备外, 系统中的流量 脉动和压力脉动都是有害的 ${ }^{[10]}$, 且二者始终以耦合 关系存在于系统中。从液压动力元件角度来看, 液 压泵工作容积发生的周期性变化使其瞬时流量是脉 动的 ${ }^{[11]}$ 。流量脉动在系统管路和负载阻抗的作用下 产生压力脉动, 同时产生波的反射、折射和干涉 ${ }^{[5]}$ 。 从液压控制元件角度来看, 在启停、换向、变速等 系统状态突变的情况下, 控制阀由于惯性作用而产 生的反向压力脉动会增大系统的脉动振幅 ${ }^{[10]}$ 。从液 压系统角度来看, 当元件和管路的固有频率与耦合 脉动的频率相同或相近时会发生共振, 共振会增大 系统的脉动和噪声、降低机械结构的寿命、影响系 统的控制性能, 严重时还会引发重大事故 ${ }^{[12]}$ 。从操 作者角度来看, 人体的骨骼和肌肉构成许多空腔和 诸如心、肺等的弹性系统, 因此振动也会引发器官 的共振, 给人体带来极大的伤害 ${ }^{[13]}$ 。此外, 振动是
噪声的主要来源, 强噪声会加速机械结构的疲劳损 伤, 甚至断裂, 也会对人的听觉组织、中枢神经系 统等造成不良影响 ${ }^{[14]}$ 。

\section{2 主动振动控制策略}

\section{1 基本原理}

主动振动控制在结构噪声控制和空气噪声控制 领域已得到广泛和成功的应用 ${ }^{[15-18]}$ 。主动振动控制 基于波的相消性干涉原理，利用次级脉动源产生与 源脉动波幅值相等、相位差 $180^{\circ}$ 的次级脉动波, 使两种波形叠加后为直线 ${ }^{[19]}$, 其原理图如图 1 所示。

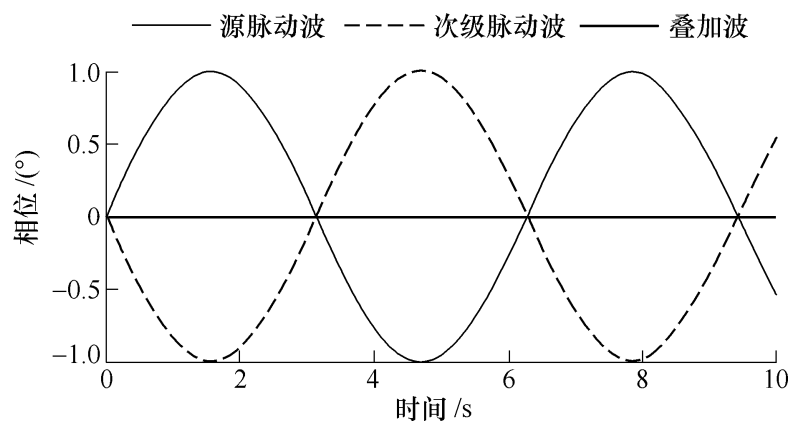

图 1 主动振动控制原理图

\section{2 系统组成}

主动控制系统主要由受控对象、作动器、控制 器、测量系统、能源等组成 ${ }^{[4,20]}$ 。对液压系统而言, 受控对象是管路系统; 常用的作动器包括液压式(如 电液伺服油缸)、电磁式(如比例电磁铁)、压电式等; 控制器多采用微处理系统, 是主动控制系统的核心 环节; 测量系统包括传感器、适调器、放大器、滤 波器等, 用于将受控对象的振动信息进行转换并传 输到控制器输入端的各个环节 ${ }^{[21]}$; 能源即补油原。

\section{3 控制方法}

根据产生次级脉动波的方式, 适用于液压系统 的主动振动控制方法可分为伺服作动筒控制、液压 阀控制和非侵入式结构控制三类 ${ }^{[22]}$ 。

第一类伺服作动筒控制是在系统主回路消振点 处串联或旁接一伺服作动筒, 通过控制此作动筒的 往复动作改变管路容积, 以此来产生能够抵消源脉 动波的次级脉动波。这种控制方法不借助流量的增 减实现脉动的衰减, 但根据牛顿第三运动定律, 作 动筒的往复动作在产生次级脉动波的同时, 管壁及 管路中的流体会对作动筒产生同等大小的流固耦合 作用反力, 这会增加实时性控制和稳定性控制的难 度, 可以考虑提高控制算法的精度或装配其他元件 来平衡管路内的压力变化。

第二类液压阀控制是在系统主回路的消振点处 旁接一高频响液压阀, 通过控制此阀的动作在主回 
路中溢流或流入适量油液, 以此来产生抵消源脉动 波的次级脉动波。

根据液压阀的工作方式, 液压阀控制可以进一 步分为分流式、入流式和分流入流式控制 ${ }^{[23]}$ 。分流 式控制的原理与溢流阀的原理相似, 即当主回路中 的脉动波处于波峰时, 通过开启高频响液压阀溢流 适量油液来降低脉动峰值; 其安装方式是将高频响 液压阀的一端接入主回路引出的一条支路上, 另一 端接回油箱, 其原理图如图 2 所示。入流式控制需 要增加补油装置, 其原理与节流阀的原理相似, 即 当主回路中的脉动波处于波谷时, 通过增大节流阀 开口流入适量油液来弥补脉动谷值; 当主回路中的 脉动波处于波峰时, 通过减小节流阀开口产生次级 脉动波来抵消源脉动波; 其安装方式是将高频响液 压阀的一端接入主回路引出的一条支路上, 另一端 接补油原的出口, 其原理图如图 3 所示。分流入流 式控制也需要增加补油装置, 其原理相当于分流式 与入流式控制原理的结合, 既能溢流适量油液来降 低脉动峰值, 又能流入适量油液来弥补脉动谷值; 其安装方式是将高频响液压阀的一端接入主回路引 出的一条支路上, 另一端接回油箱以及补油原的出 口，其原理图如图 4 所示。

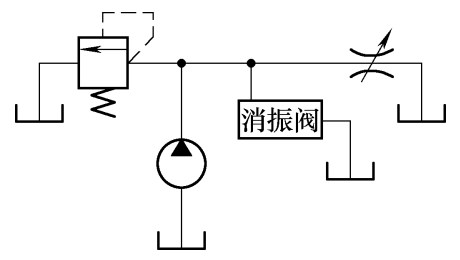

图 2 分流式主动脉动控制原理图

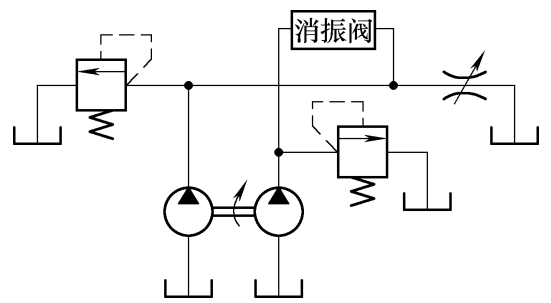

图 3 入流式主动脉动控制原理图

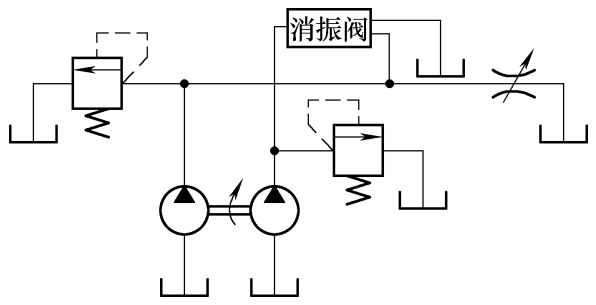

图 4 分流入流式主动脉动控制原理图

这三种控制方法中, 分流式控制的装置最简单， 但需溢流一定高压油, 因此会带来一定程度的能量 损失; 入流式控制和分流入流式控制都需要补油装 置, 因此结构更复杂、控制难度更高, 且研究程度
偏低; 其中脉动衰减效果最好的是分流入流式控制。

第三类非侵入式结构控制是在系统主回路消振 点处的管壁外安装固定一个或多个一般由固定支 架、驱动材料、单自由度物体、线圈、传感器等构 成的非侵入式主动作动器, 通过控制驱动材料的变 形带动质量块、活塞等结构对管路施加压力, 以此 来产生抵消源脉动波的次级脉动波。该控制方式一 般用于低压液压系统中，能耗较低、操作灵活、响 应较快, 但控制精度和稳定性较差, 容易受到外界 环境的影响。

\section{4 控制算法}

液压系统一般具有较高的非线性和不确定的动 力学特性, 简单的线性或非线性微分方程不能充分 表达相应的实际系统，因此基于精确模型的控制算 法并不适用。在液压系统流体脉动主动控制中常用 的控制算法有如下四种。

一是自适应控制算法, 它适合具有一定不确定 性的系统，在分析和处理的过程中能够根据数据特 征自动调整模型参数, 最终以最佳的状态逼近目标。 其中最常用的是自适应前馈 $\mathrm{x}$ 滤波最小均方 (Filtered-x least mean square, FxLMS)算法, 该算法 容易实现、运算较快, 但是对控制通道敏感, 鲁棒 性相对较差 ${ }^{[24]}$ 。

二是自寻最优控制算法, 它适合无精确数学模 型但具有非线性特性的受控系统，在控制过程中通 过不断测量、理解、计算、判断等可以得到实时的 最优状态，其控制参数在优化过程中能随时被修正 并稳定地作用于系统，具有优异的自适应性 ${ }^{[25-26]}$ 。 该算法适用于管路非线性和负载刚度特性对系统影 响较大的液压系统的流体脉动主动控制中。

三是神经网络控制算法, 它不要求对系统进行 透彻的了解, 通过一定量的输入输出样本进行学习 和训练, 就能够以极高精度逼近任何复杂的非线性 映射, 具有很强的自学习能力、自适应能力和非线 性映射能力 ${ }^{[27-29]}$ 。

四是模糊控制算法, 它适合已知信息不精确 或不确定的复杂非线性系统 ${ }^{[30]}$, 依据理论和经验 分析总结出控制规则, 再通过基于该规则的合理 推理去控制系统，具有很强的鲁棒性 ${ }^{[31]}$ 。该算法 适用于需要快速响应的液压系统的流体脉动主动 控制中 ${ }^{[32]}$ 。

\section{3 流体脉动主动控制研究现状}

\section{1 伺服作动筒主动控制}

1990 年, 日本神奈川大学的 KOJIMA 等 ${ }^{[33]}$ 在 
液压管路系统脉动前进波流速的实时性测量方法研 究中, 搭建了脉动主动控制的试验平台。他们将伺 服作动筒旁接在主回路消振点处引出的一条支路上 作为次级脉动源, 将两枚压力传感器安装在伺服作 动筒上游的量测段管壁上。根据平面波理论, 从压 力和速度信号中获得流体脉动的前进波成分, 并用 数字信号处理器等进行实时的高速计算。

1996 年, YAMAOKA 等 ${ }^{\left[{ }^{[4]}\right.}$ 对液压系统流体脉
动的主动衰减开展了更加深入的研究。他们先后将 简谐振动波和宽频带随机振动波作为源脉动波, 利 用补油洜和伺服作动筒的往复动作产生用以抵消源 脉动波的次级脉动波; 将两枚压力传感器分别安装 在伺服作动筒的上游和下游管壁上, 利用二者的压 差信号作为控制器的输入, 其原理图如图 5 所示。 采用 FxLMS 算法, 在 $10 \sim 800 \mathrm{~Hz}$ 频域内取得了至 少 $20 \mathrm{~dB}$ 的衰减效果。

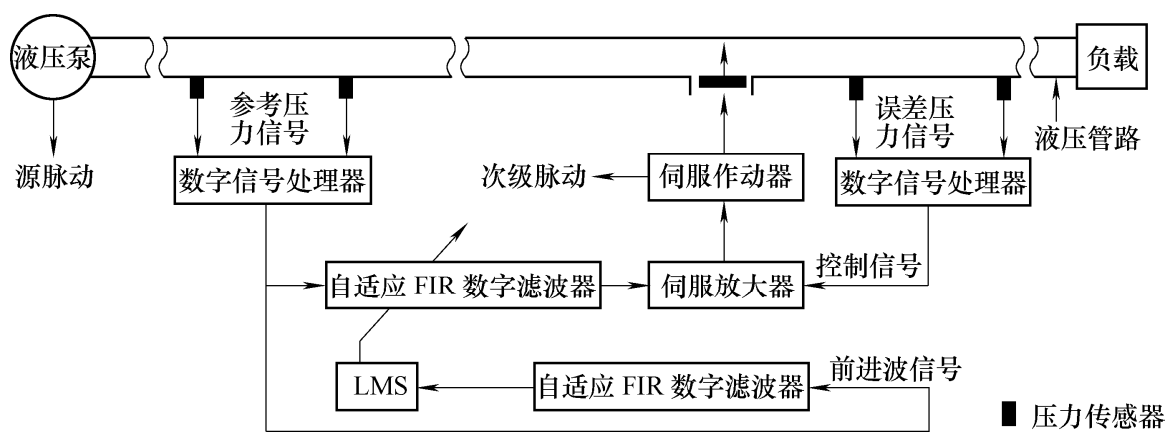

图 5 伺服作动筒主动消振原理图

2013-2014 年, 英国巴斯大学的 JOHNSTON 等 ${ }^{[35]}$ 提出了用于估计管路系统中非定常流量的方 法, 将基于波传播模型的压力信号作为边界条件来 估计非定常流量, 并通过试验验证了此方法的鲁棒 性和可靠性。利用消振点上游采集到的多个压力信 号作为控制器的输入信号, 采用有限差分近似方法 来实时估计上游脉动波波形, 取得了 $10 \mathrm{~dB}$ 的衰减 效果。这种思路可对 KOJIMA 等 ${ }^{[33-34]}$ 采用的方法进 行优化, 降低脉动衰减效果与系统稳定性对实时前 进波精度的依赖性。

1996 年, 日本东京工业大学的 YOKOTA 等 ${ }^{[36]}$ 设计了一种由叠层压电陶瓷(Piezoelectric transducer, PZT)直驱活塞、用于降低压力平衡容腔内阻抗的被 动液压蓄能器等组成的主动液压蓄能器, 并将其串 联在系统的泵源附近。利用安装在泵前的光电传感 器采集到的泵转速信号作为参考压力信号, 利用消 振点后的压力传感器信号作为误差信号, 并将两种 信号传输给微处理系统。采用 FxLMS 算法, 通过 驱动 $\mathrm{PZT}$ 来控制与流体流速成比例的活塞的运动速 度, 利用活塞往复运动产生的次级脉动波来消减源 脉动波, 同时减少脉动压力波向管路下游的传播, 其原理图如图 6 所示。试验证实了脉动波在 500 $1000 \mathrm{~Hz}$ 频域内被有效衰减。

目前, 国内外学者对应用在液压系统中的伺服 作动筒主动消振方法的研究比较少, 且近年来并无 突出进展, 原因是活塞在介质中往复动作产生的用 来抵消源脉动波的次级脉动波并不可靠。在通过活 塞产生次级脉动波的同时, 管壁及管路中的流体会
对它产生同等大小的流固耦合作用反力, 而活塞的 往复动作也可能形成系统不需要的压力差。由于活 塞具有自由度, 这些力作用在活塞上可能会影响它 的运动趋势, 进而影响次级脉动波波形。因此, 伺 服作动筒主动消振方法对控制系统的实时性、自适 应性、稳定性、干扰补偿能力等都有非常高的要求, 对于方法的改进与突破尚具有一定的局限性。

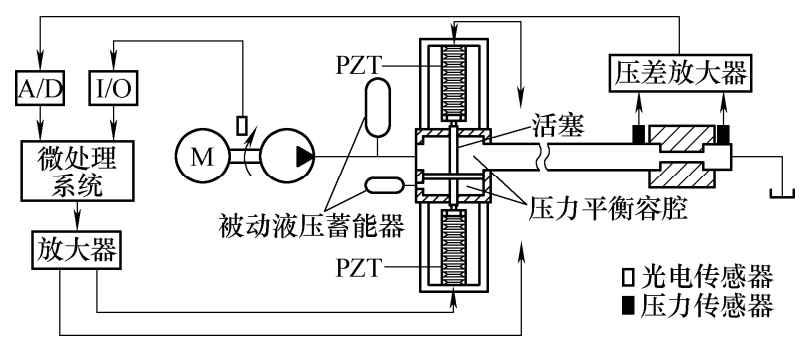

图 6 主动液压蓄能器消振原理图

\section{2 液压阀主动控制}

被动消振方法对降低低频脉动的效果并不理 想, 2001 年, 西安交通大学的邢科礼等 ${ }^{[32]}$ 采用分流 式主动控制方法对低频脉动衰减展开了研究。他们 利用一个电液伺服阀产生的次级脉动波去消除另一 个电液伺服阀产生的源脉动波, 并且对比了两种控 制算法的优劣。试验结果显示, 相比 FxLMS 算法, 神经网络算法能更好地保证数据采集过程中的实时 性和收玫性。在 20 70 Hz 的频域范围内, 源脉动 波形为正弦波、方波和三角波时, 主动消振法均能 适应性地实现 8.7 $11 \mathrm{~dB}$ 的脉动衰减。

2002 年, 韩国首尔国立大学的 KIM 等 ${ }^{[37]}$ 设计 了一种新型主被动复合控制系统, 在扩张腔式被动 
衰减器上额外引出 2 个端口, 用来连接主动控制系 统。采用模糊控制算法, 利用一个高频响伺服阀控 制一组插装阀的先导压力, 进而由插装阀组控制蓄 能器在极短时间内释放出过多流量以衰减脉动峰 值, 其原理图如图 7 所示。其中, 插装阀的设计尺 寸决定了系统是否具有快速响应冲击并释放大流量 油液的能力。试验结果显示, 设计的插装阀和主动 控制方法适用于高速冲击和大流量系统, 能够在 5 20 ms 内降低 40\%峰值压力。

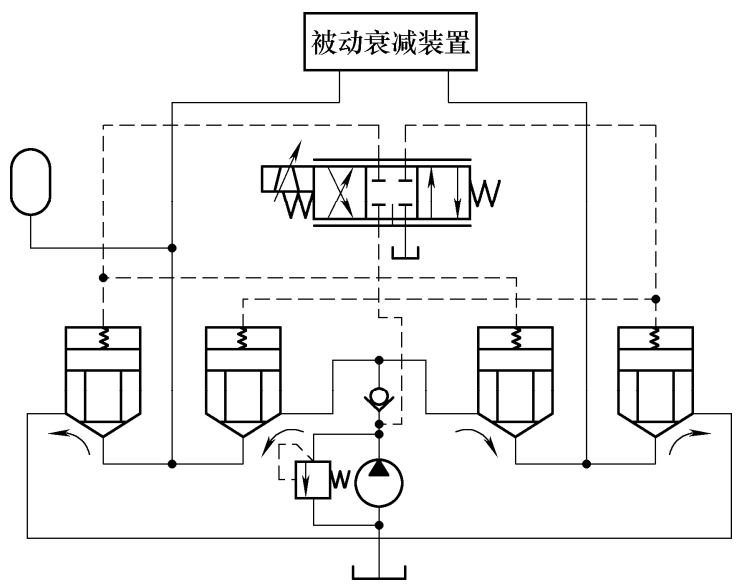

图 7 主被动复合控制原理图

2003 年, 北京航天大学的焦宗夏等 ${ }^{[38]}$ 设计了一 种叠层压电直驱式阻尼阀 (Piezoelectric orifice valve, POV), 采用分流式主动控制方法将其旁接于 泵源附近作消振阀。采用自寻最优控制算法, 利用 电压信号调节阀的开口大小，进而调节次级脉动波 波形, 其原理图如图 8 所示。试验结果显示, 在 7 柱塞百转速 $3000 \mathrm{r} / \mathrm{min}$ 的条件下, 当消振频率为 350 $\mathrm{Hz}$ 时, 该主动消振系统能够衰减 68\%的脉动压力。 文章研究了未知振幅和相位的单一频率点的消振问 题，为分布式消振提供了理论和实践基础。

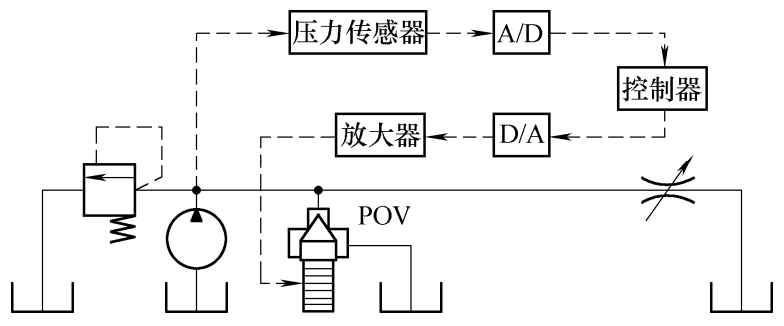

图 8 压电直驱式阻尼阀主动脉动控制原理图

飞机在滑行、爬升、巡航、机动等不同的飞行 阶段中, 管路中流体脉动频率会在较宽的范围内波 动。由于被动消振方法对低频脉动的衰减效果不佳, 且不具备自适应能力, 因此不适用于衰减飞机液压 能源管路系统的耦合脉动。而主动消振方法通常为 单点消振, 存在寻找误差传感器最佳布局位置困难 而引起脉动衰减效果不理想的问题。2007 年, 北京
航空航天大学的欧阳平超等 ${ }^{[39]}$ 提出了用在飞机液 压管路上的分布式流体脉动主动控制方法，即沿管 路不同位置布置多个压电陶瓷直驱伺服阀和误差压 力传感器, 以实现整条管路的振动控制。在 9 柱塞 泵转速为 $600 \mathrm{r} / \mathrm{min}$ 、管路内流体压力为 $5 \mathrm{MPa}$ ，以 及采样频率为 $2 \mathrm{kHz}$ 的条件下，采用 FxLMS 算法, 当目标消振基频为 $90 \mathrm{~Hz}$ 时, 试验取得了 $10 \mathrm{~dB}$ 以 上的衰减效果。

2008 年，英国巴斯大学的 WAND 等 ${ }^{[00]}$ 发现来 自液压回路两端的反射波影响了消振方法的可靠性 和稳定性，因此，他们以伺服阀作为消振阀，采用 FxLMS 算法, 研究了一般液压系统中由泵引起的多 次谐波脉动的主动振动控制问题。采用在线次级路 径识别方法, 实现了在没有关于液压系统性能先验 知识的情况下可以进行主动消振, 但为该在线方法 施加到系统中的辅助白噪声信号可能带来残留噪 声。使用特征方法(Method of characteristics，MOC) 搭建系统动态特性模型并进行仿真分析, 经试验验 证，该方法在液压系统的一般工况下能够取得约 20 $\mathrm{dB}$ 的脉动衰减效果。

在利用液压阀的主动控制中，阀的频响特性是 影响控制性能的关键因素。2011 年, 英国巴斯大学 的 BRANSON 等 ${ }^{[41]}$ 设计了一种高频响、宽带宽的 压电伺服阀。压电作动器直接作用于阀门, 使其能 够在 $1.5 \mathrm{~ms}$ 内完全打开或关闭, 实现了阀的快速动 态响应。采用多边阀口，允许在低压降和小位移时 通过大流量, 此阀能够在 $2 \mathrm{MPa}$ 的压降下通过 65 $\mathrm{L} / \mathrm{min}$ 的流量。传统电液伺服阀和高频响比例阀的 工作频带宽一般是 150 300 Hz，此阀的工作频带 宽能够达到 425 $2000 \mathrm{~Hz}$ 。2015 年，英国巴斯大 学的 SELL 等 ${ }^{[42]}$ 设计了一种应用于数字液压回路的 高频响、大流量液压阀, 能够在 $0.5 \mathrm{~ms}$ 内快速开启, 在 $1 \mathrm{MPa}$ 的压降下通过大于 $50 \mathrm{~L} / \mathrm{min}$ 的流量。目前, 该阀的驱动力来自一组恒压源, 还需要更加高效便 捷的驱动方法进行更新。

2014 年, 北京航空航天大学的官长斌等 ${ }^{[43]}$ 设计 了一种压电直驱滑阀(Piezoelectric direct-drive slide valve, PDDSV), 采用分流式主动控制方法, 将 PDDSV 的 2 个油口旁接在泵出口附近的管路上, 另 2 个油口接回油箱。采用自寻最优控制算法, 通过 PDDSV 阀肩的双侧溢流，阀芯运动一个周期所产生 的次级溢流波可以消除 2 个周期的源脉动波，这使 得 PDDSV 在 $1 / 2$ 压力脉动频率下就可以进行消振。 因此, PDDSV 适用于高速液压管路系统, 其原理图 如图 9 所示。系统脉动中通常包含基波和多次谐波， 对所有谐波进行滤波是十分困难的。脉动的能量主 
要集中在基波和二次谐波上, 因此只要衰减掉主要 脉动成分, 液压系统的脉动水平就会大大降低 ${ }^{[10]}$ 。 试验结果显示, 在 9 柱塞原转速为 $1200 \mathrm{r} / \mathrm{min}$ 、管 路内流体压力为 $9 \mathrm{MPa}$, 以及采样频率为 $2 \mathrm{kHz}$ 的 条件下, 当 PDDSV 的工作频率为 $1 / 2$ 基频频率时, 可消除基波 42.3\%的压力脉动; 当 PDDSV 的工作 频率为 $1 / 2$ 二次谐波频率时, 可消除二次谐波 $26.7 \%$ 的压力脉动; 当 PDDSV 用于混合波的脉动衰减时, 可实现 $63.3 \%$ 的脉动衰减效果。

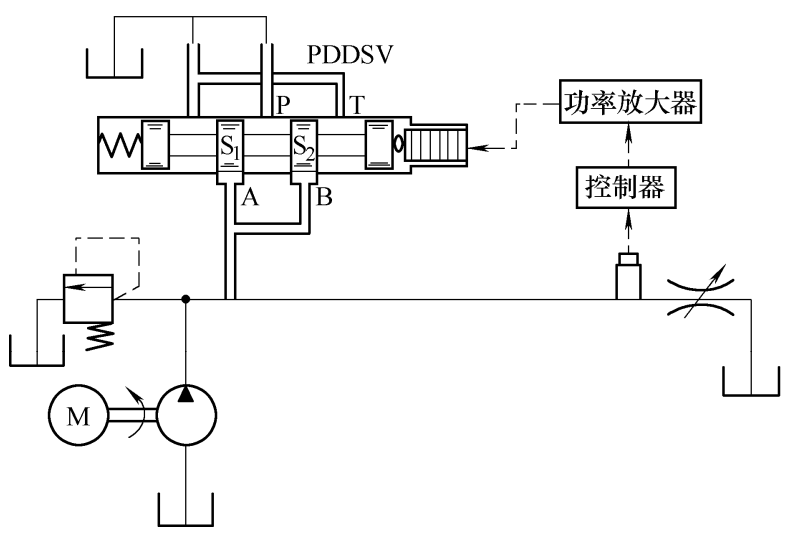

图 9 压电直驱滑阀主动脉动控制原理图

2016 年, 北京航空航天大学的 ZHENG 等 ${ }^{[44]}$ 提出用有效梯度算法来优化控制参数的流体脉动 主动消振方法, 其中被优化的参数有脉动幅值、频 率和相位。采用分流式主动控制方法, 将一快速伺 服阀旁接于管路中产生次级脉动波。在 9 柱塞泵转 速为 $1200 \mathrm{r} / \mathrm{min}$ 、管路内流体压力为 $5.1 \mathrm{MPa}$, 以 及采样频率为 $2 \mathrm{kHz}$ 的条件下, 目标消振频率为 $200 \mathrm{~Hz}$ 时, 试验取得 35.6\%的脉动衰减效果。但 目前该主动消振方法持续衰减脉动的时长并不 长, 因此需要提高此算法的稳定性以适应不同的 工况。

2016-2017 年, 西南交通大学的季晓伟等 ${ }^{[45-46]}$ 对入流式有源脉动主动控制进行了研究, 设计了一 种高频响的叠层 PZT 直驱型节流阀作为消振阀, 采 用神经网络算法, 通过补油䈋和消振阀为系统弥补 脉动谷值。当目标消振频率为 $90 \mathrm{~Hz}$ 和 $180 \mathrm{~Hz}$ 时, 试验均获得了 $67 \%$ 的脉动衰减效果, 其原理图如图 10 所示。

三种液压阀控制方法均是将消振阀旁接于系统 中, 但并未说明选择旁接而非串联的原因。2014 年, 英国巴斯大学的 PAN 等 ${ }^{[47]}$ 对比研究了串联消振方 法和旁接消振方法的脉动衰减效果, 证明了后者是 更实用、有效的消振方法。串联消振方法通过将消 振阀接入系统管路与负载之间来产生可控的脉动压 差, 消振阀连接方式如图 11 所示; 旁接消振方法即

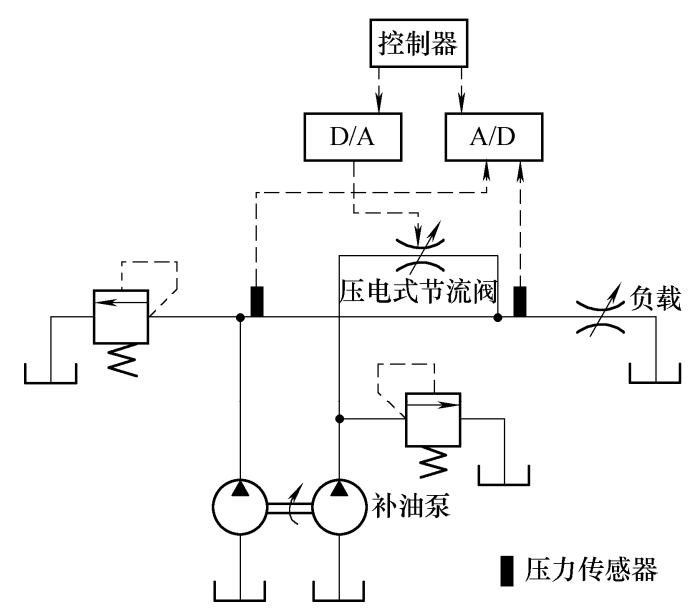

图 10 入流式主动控制原理图

分流式控制, 通过向管路中溢流流量来产生次级脉 动波, 消振阀连接方式如图 12 所示。试验结果表明 串联消振效果对流入消振阀的流量大小有相当大的 依赖性, 而且引入白噪声带来的影响也大于旁接消 振。更进一步考虑, 构成旁接消振回路的条件易于 实现, 分别是宽带宽、高压差、低流速; 而构成串 联消振回路的条件是宽带宽、低压差、高流速, 意 味着需要一个尺寸更大、造价更高的特制液压阀代 替一般消振阀。

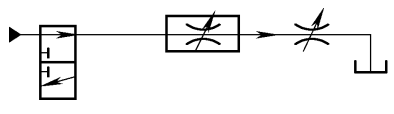

图 11 串联结构

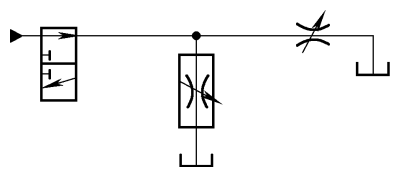

图 12 旁接结构
2017 年, PAN 等 ${ }^{[9]}$ 首次设计了针对解决液压系统 降压转换器带来严重脉动问题的控制器, 采用 FxLMS 算法, 将谐波设定为 $40 \mathrm{~Hz} 、 80 \mathrm{~Hz}$ 和 $120 \mathrm{~Hz}$, 基于 串联消振结构和并联消振结构的试验结果均显示, 采用该控制器能够取得超过 $30 \mathrm{~dB}$ 的脉动衰减效果。

\section{3 非侵入式结构控制}

1996 年, 英国南安普顿大学的 BRENNAN 等 ${ }^{[48]}$ 设计了一种用于管路系统流体脉动衰减的非侵入式 磁致伸缩主动作动器(长 $88 \mathrm{~mm}$ ), 如图 13 所示, 将 其固定在一段充满水的有机玻璃管外壁上, 将该整 体作为主动控制装置串接在管路系统中, 采用 FxLMS 算法, 通过改变磁致伸缩材料的磁化状态来 带动活塞运动, 进而将作用力施加给管壁以产生抵 消源脉动波的次级脉动波。试验结果显示, 这种方 法能够在 10 1 $000 \mathrm{~Hz}$ 频段内实现 $20 \mathrm{~dB}$ 的脉动衰 减, 但与此同时也会增大消振点附近管壁的振动。 此外, 在使用一个主动作动器的情况下, 主动作动 器与流体压力之间的耦合作用力会导致其实现有效 脉动衰减的频率点较少, 因此作者建议使用 2 个主 
动作动器以达到更好的脉动衰减效果。

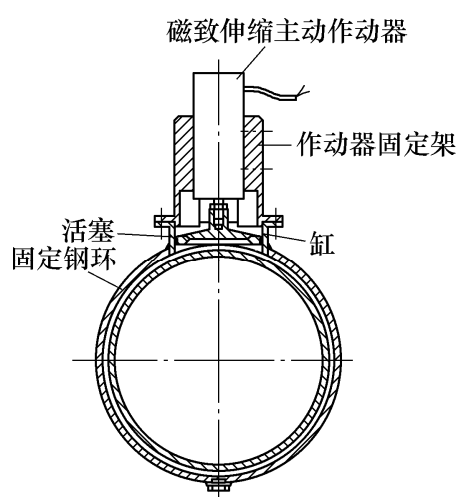

图 13 非侵入式磁致伸缩主动作动器结构简图

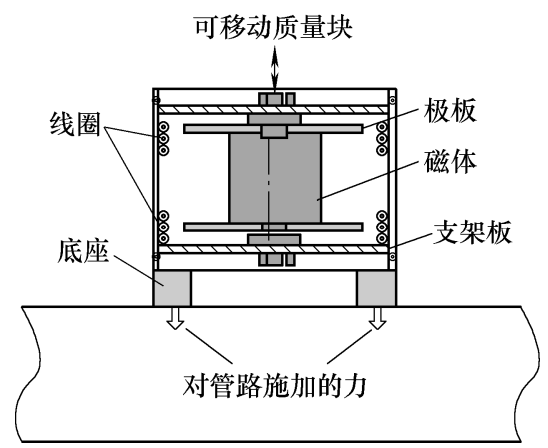

图 14 非侵入式磁致伸缩主动作动器结构简图

压电叠层驱动器具有重量轻、驱动力大、驱动 位移小、工作频率范围宽、响应速度快等优点, 是 结构主动振动控制的理想执行机构 ${ }^{[49]}$ 。

1998 年, 瑞典龙讷比卡尔斯克鲁纳大学的 MAILLARD ${ }^{[50]}$ 设计了一种由一段短管和环形主动 作动器组成的非侵入式环形主动消振装置。其中, 短管为与两端联接管路直径相同但壁厚相对较薄的 $150 \mathrm{~mm}$ 长的钢管; 环形主动作动器以环抱挤压的方 式安装在短管中间的外管壁上，由 6 个 PZT 叠层、 钢制环形固定支架、压力传感器、控制器等组成, 如图 15 所示。采用 FxLMS 算法, 将 PZT 逆压电效 应产生的合力作用于管路上, 以此产生次级脉动波。 试验结果显示, 对每个 PZT 叠层施加约 $3500 \mathrm{~N}$ 预 加力、对主动作动器施加 $8 \mathrm{~N} \cdot \mathrm{m}$ 预加扭矩时, 在 流体静压力保持在 $2 \mathrm{MPa}$ 左右的条件下, 能够在 0

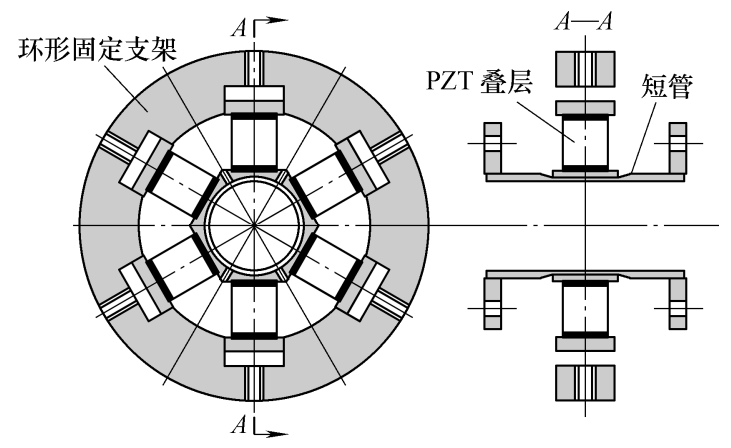

图 15 非侵入式环形主动作动器结构简图
$600 \mathrm{~Hz}$ 频段内实现 6 15 dB 的压力脉动衰减, 试 验系统原理图如图 16 所示。该主动消振装置结构紧 凑、便于安装, 但该研究的主要目标是结构设计和 性能测试, 因此研究过程存在液压系统压力等级偏 低、PZT 叠层到管壁的能量损失较大、PZT 温升影 响其应变位移、控制精度不足等缺点。

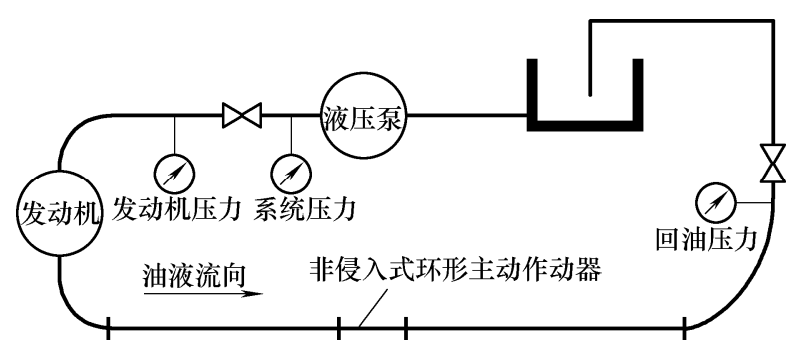

图 16 试验系统原理图

1999 年，法国巴黎建筑科学技术中心的 MAILLARD 等 ${ }^{[51]}$ 将其设计的非侵入式环状 PZT 叠 层主动作动器应用于约 $7 \mathrm{kPa}$ 的低压流体管路系统 中, 试验取得一定效果, 并分析了该主动作动器应 用于液压发动机排放管上的振动控制性能。

2002 年，美国弗吉尼亚理工大学的 KIYAR 等 ${ }^{[52]}$ 对管路系统中采用非侵入式结构主动作动器 的主动控制方法展开了研究。研究表明, 管道的弯 曲和不连续会将不同类型的振动波耦合在一起。作 者将 3 个如图 14 所示的磁致伸缩主动作动器固定在 管路上，采用 FxLMS 算法来控制电磁作动器的动 作。试验中给定 3 个作动器不同的输入功率, 结果 显示它们可以分别衰减不同类型的振动波。2 个压 力传感器采集参考压力信号保持不变, 同时分别将 6 个加速度传感器、 2 个压力传感器和全部 8 个传感 器作为误差传感器进行试验, 在 $50 \sim 450 \mathrm{~Hz}$ 的频域 范围内分别得到了壳体波约 $8.2 \mathrm{~dB}$ 及流体波约 $1 \mathrm{~dB}$ 的衰减、流体波约 $4 \mathrm{~dB}$ 的衰减(壳体波未受影响, 某些类型的振动波甚至得到了增强)、壳体波约 8.25 $\mathrm{dB}$ 的衰减及流体波约 $6.4 \mathrm{~dB}$ 的衰减效果。试验结 果表明, 传感器与主动作动器的组合方式及其安装 位置都会对脉动衰减效果产生影响, 并且在管路系 统中应用多个非侵入式主动作动器可以对多种类型 的振动波进行衰减, 这也从理论层面证实了 BRENNAN 等 ${ }^{[48]}$ 的想法。

2015 年，英国南安普顿大学的 CHEER 等 ${ }^{[53]}$ 基 于文献[50]的研究进行了如下改进。他们使用 8 个 PZT 叠层以增强脉动控制效果; 不改变短管壁厚以 减小设计难度、增强通用性; 以独立驱动 PZT 叠层 代替同步驱动所有 PZT 叠层, 从而实现了更高阶模 态的控制, 且避免了因每个 PZT 叠层预加力的不同 而对其控制性能的影响。试验结果显示，在采样频 
率为 $2.2 \mathrm{kHz}$ 的条件下, 在 $0 \sim 1000 \mathrm{~Hz}$ 频域范围内 的平均脉动衰减量为 $12.8 \mathrm{~dB}$, 脉动衰减峰值为 30 $\mathrm{dB}$; 在 $600 \sim 800 \mathrm{~Hz}$ 频域范围内能够达到 $20 \mathrm{~dB}$ 的 衰减效果。相比文献[50], 该研究在结构设计、控 制算法、衰减频域、衰减效果上均有一定提高。

\section{4 总结与展望}

从上述分析可以看出, 目前应用和研究最多的 主动消振方法是液压阀入流式主动控制方法。它无 需补油装置, 操作相对简易, 控制难度相对较低, 但溢流会带来一定的能量损失。主动消振方法多集 中于单点消振, 即在管路某一处设置消振元件。但 是在整个管路系统中寻找最佳消振点是比较困难 的, 尤其是对于大型液压系统。换言之, 对有源脉 动系统的主动消振通常是非最佳状态的局部消振, 而消振位置选择不佳会直接影响脉动衰减的效果。 由于管路是分布式参数系统, 可以采用分布式主动 控制方法进行消振, 即沿管路不同位置布置多个 主动消振元件和压力传感器, 更全面有效地对整 个系统管路进行脉动衰减。定位最佳消振点的方 法和分布式消振法都是流体脉动主动控制未来的 研究方向。

对于有源液压系统而言, 其㬌源脉动频率往往 高达数百赫兹, 所配套的主动控制系统需要带有分 辨率高且工作频带宽的高频响消振阀。近年来, 压 电材料因其高频响、高能量密度、低能耗、小体积 等优点被广泛用作液压阀中的执行器, 以提高液压 阀的性能。但压电材料的特性往往会受到温度、湿 度、压力等外界因素的影响, 所以对材料的掌控程 度决定了其在液压系统流体脉动主动消振中的应用 程度, 对其应用特性的研究会推动主动消振方法的 进步。此外, 对新型液压阀驱动材料的研究也是未 来的研究方向。

从衰减频段来看, 被动消振普遍针对中、高频 段的脉动衰减, 对低频段的脉动衰减效果差。主动 消振基本可以实现 $0 \sim 1000 \mathrm{~Hz}$ 频域内的有效脉动 衰减, 只是不同的主动消振方法的作用频段和衰减 效果不同。主动消振具有参数自适应性, 适用于工 况多变、对性能要求高的场合, 但其能耗较高、成 本较高、控制难度较高。而被动消振虽不具备上述 主动消振的优点, 但其性能稳定、可靠性高。主被 动联合消振将成为未来的研究方向, 通过二者的优 势互补, 将用于液压系统流体脉动的衰减装置发展 成为结构简单、通用性强、自适应性强、衰减频带 宽且可靠性高的一体式元件。

\section{参 考 文 献}

[1] BAKER B B, CCPSON E T. The mathematical theory of Huygens' principle[M]. Chelsea: American Mathematical Soc., 2003.

[2] HANSEN C H, SNYDER S D, CLARK R L. Active control of noise and vibration[J]. Journal of Vibration \& Acoustics, 1996, 109(3): 9-11.

[3] PAUL L. Process of silencing sound oscillations, US2043416[P]. 1936-09-06.

[4] 顾仲权, 马扣根, 陈卫东. 振动主动控制 [M]. 北京: 国防工业出版社, 1997.

GU Zhongquan, MA Kougen, CHEN Weidong. Active vibration control [M]. Beijing: National Defence Industry Press, 1997.

[5] 曾祥荣. 液压噪声控制[M]. 哈尔滨: 哈尔滨工业大学 出版社, 1988.

ZENG Xiangrong. Hydraulic noise control [M]. Harbin: Harbin Institute of Technology Press, 1988.

[6] 周文. 主动振动控制技术的发展和应用[J]. 液压气动 与密封, 2003(4): 24-27.

ZHOU Wen. Development and application of active vibration control technology[J]. Hydraulic Pneumatics and Sealing, 2003(4): 24-27.

[7] 李树立, 焦宗夏. 液压流体脉动主动控制研究现状与展 望[J]. 机床与液压, 2006(9): 243-246.

LI Shuli, JIAO Zongxia. Research actuality and prospect of active control of hydraulic fluid fluctuation[J]. Machine Tool \& Hydraulics, 2006(9): 243-246.

[8] SHANG Xia, ZHOU Hua, XIE Anhuan, et al. Filtering characteristics of string hydraulic pulsation attenuator[C]// ASME 2017 Dynamic Systems and Control Conference. American Society of Mechanical Engineers, 2017 : V003T22A004.

[9] PAN M. Adaptive control of a piezoelectric valve for fluid-borne noise reduction in a hydraulic buck converter[J]. Journal of Dynamic Systems Measurement \& Control, 2017, 139(8): 081007.

[10] 陈季萍. 降低液压系统压力脉动方法的研究[J]. 煤矿 机械, 2006, 27(6): 955-957.

CHEN Jiping. Research in approaches to decrease pressure pulsation of hydraulic system[J]. Coal Mine Machinery, 2006, 27(6): 955-957.

[11] 李壮云. 液压元件与系统[M]. 北京: 机械工业出版社, 2011.

LI Zhuangyun. Hydraulic components and systems[M]. Beijing: Mechanical Industry Press, 2011.

[12] 郭长虹, 史俊强, 权凌霄, 等. 航空液压洜流量脉动吸 收的新途径一一仿生式液压管路[C]// 全国流体传动与 
控制学术会议, 2016.

GUO Changhong, SHI Junqiang, QUAN Lingxiao, et al. A new method of fluid pulsation absorption of aero hydraulic pump-bionic hydraulic pipeline [C]// National Conference on Fluid Power Transmission and Control, 2016.

[13］周新祥. 噪声控制技术及其新进展[M]. 北京：冶金工 业出版社, 2007.

ZHOU Xinxiang. Noise control technology and its new progress[M]. Beijing: Metallurgical Industry Press, 2007.

[14] 张恩慧, 殷金英, 邢书仁. 噪声与振动控制 $[\mathrm{M}]$. 北京: 治金工业出版社, 2012.

ZHANG Enhui, YIN Jinying, XING Shuren. Noise and vibration control[M]. Beijing: Metallurgical Industry Press, 2012.

[15] BAO C, SAS P, BRUSSEL H V. Adaptive active control of noise in 3-D reverberant enclosures[J]. Journal of Sound \& Vibration, 1993, 161(3): 501-514.

[16] KUO S M, MORGAN D. Active noise control systems: algorithms and DSP implementations[M]. New York: John Wiley \& Sons, Inc., 1995: 1071-1074.

[17] ZHANG M, LAN H, SER W. Cross-updated active noise control system with online secondary path modeling[J]. IEEE Transactions on Speech and Audio Processing, 2001, 9(5): 598-602.

[18] HILliS A J, HARRISON A J L, STOTEN D P. A comparison of two adaptive algorithms for the control of active engine mounts[J]. Journal of Sound \& Vibration, 2005, 286(1): 37-54.

[19] KOJIMA E, SHINADA M, YAMAOKA T. Development of an active attenuator for pressure pulsation in liquid piping systems: trial construction of the system and fundamental experiments on attenuation characteristics[J]. JSME International Journal Series B Fluids and Thermal Engineering, 1993, 36(2): 230-237.

[20] 李宏男. 结构振动与控制 $[M]$. 北京: 中国建筑工业出 版社, 2005.

LI Hongnan. Structural vibration and control[M]. Beijing: China Building Industry Press, 2005.

[21] 周星德, 姜冬菊. 结构振动主动控制[M]. 北京: 科学 出版社, 2009 .

ZHOU Xingde, JIANG Dongju. Active control of structural vibration[M]. Beijing: Science Press, 2009.

[22] 季晓伟. 流体脉动的入流式主动控制[D]. 成都: 西南交 通大学, 2017.

JI Xiaowei. Inflow active control of fluid pulsation[D].
Chengdu: Southwest Jiaotong University, 2017.

[23] 邢科礼. 液压系统压力脉动的衰减理论及方法研究 [D]. 西安：西安交通大学, 1998.

XING Keli. Research on attenuation theory and method of pressure pulsation in hydraulic system[D]. Xi'an: Xi'an Jiaotong University, 1998.

[24] 汪光森, 王乘. 自适应主动噪声控制系统的仿真研究 [J]. 电声技术，2004(8): 57-59.

WANG Guangsen, WANG Cheng. Simulation of adaptive active noise control system[J]. Audio Engineering, 2004(8): 57-59.

[25] 谢永斌, 罗忠, 胡保生. 自寻最优控制原理及其方法 [J]. 西安工业学院学报，1996(2): 100-104.

XIE Yongbin, LUO Zhong, HU Baosheng. The principle and the method of a self-optimal control[J]. Journal of Xi'an Institute of Technology， 1996(2): 100-104.

[26] 朱晓锦, 陶宝祺. 基于自寻最优控制方法实现结构振动 主动控制[J]. 振动.测试与诊断, 1999(1): 15-19.

ZHU Xiaojin, TAO Baoqi. Structure active vibration control based on adaptive searching optimazation[J]. Journal of Vibration Measurement \& Diagnosis, 1999(1): 15-19.

[27] HIDAKA S, AHN Y K, MORISHITA S. Adaptive vibration control by a variable-damping dynamic absorber using ER fluid[J]. Journal of Vibration \& Acoustics, 1999, 121(3): 373-378.

[28] HINTON G E, OSINDERO S, TEH Y W. A fast learning algorithm for deep belief nets[J]. Neural Computation, 2006, 18(7): 1527-1554.

[29] O’SHEA T, HOYDIS J. An introduction to deep learning for the physical layer[J]. IEEE Transactions on Cognitive Communications and Networking, 2017, 3(4): 563-575.

[30] SHAO J, CHEN L, SUN Z. The application of fuzzy control strategy in electro-hydraulic servo system[C]// IEEE International Conference Mechatronics and Automation, IEEE, 2005, 4: 2010-2016.

[31] 胡海岩, 郭大蕾, 翁建生. 振动半主动控制技术的进 展 $[\mathrm{J}]$. 振动・测试与诊断, 2001(4): 235-244.

HU Haiyan, GUO Dalei, WENG Jiansheng. Recent advances in semi-active control of vibration[J]. Journal of Vibration Measurement \& Diagnosis, 2001(4): 235-244.

[32] 邢科礼, 葛思华, 丁崇生. 基于神经网络的有源压力脉 动衰减的试验研究 [J]. 液压气动与密封, 2001(2): 2-4. XING Keli, GE Sihua, DING Chongsheng. Experimental study of active attenuator for pressure pulsation using a neural network[J]. Hydraulic Pneumatics and Sealing, 
2001(2): 2-4.

[33] KOJIMA E, SHINADA M. Development of an active attenuator for pressure pulsation in liquid piping systems; 1st report, a real time measuring method of progressive wave in a pipe[J]. Transactions of the Japan Society of Mechanical Engineers Series B, 1990 , 56(530) : 2937-2944.

[34] YAMAOKA T, KOJIMA E. Development of an activeadaptive attenuator for pressure pulsation in liquid piping systems[J]. Proceedings of the JFPS International Symposium on Fluid Power, 1996, 1996(3): 449-454.

[35] JOHNSTON N, PAN M, KUDZMA S, et al. Use of pipeline wave propagation model for measuring unsteady flow rate[J]. Journal of Fluids Engineering, 2014, 136(3): 031203.

[36] YOKATA S, SOMADA H, YAMAGUCHI H. Study on an active accumulator. (Active control of high-frequency pulsation of flow rate in hydraulic systems) [J]. Bulletin of the JSME, 1996, 39(1): 119-124.

[37] KIM D H, PARK J W, LEE G S, et al. Active impact control system design with a hydraulic damper[J]. Journal of Sound and Vibration, 2002, 250(3): 485-501.

[38] JIAO Z, CHEN P, WANG S. Adaptive vibration active control of fluid pressure pulsations[J]. Proceedings of the Institution of Mechanical Engineers Part I Journal of Systems \& Control Engineering, 2003, 217(4): 311-318.

[39] 欧阳平超, 焦宗夏, 刘红梅. 分布式液压流体脉动主动 控制方法[J]. 北京航空航天大学学报, 2007, 33(9): 1060-1063.

OUYANG Pingchao, JIAO Zongxia, LIU Hongmei. Study on distributed active control of fluid pulsation in hydraulic piping[J]. Journal of Beijing University of Aeronautics \& Astronautics. 2007, 33(9): 1060-1063.

[40] WANG L, JOHNSTON D N. Adaptive attenuation of narrow band fluid borne noise in a simple hydraulic system[C]//Bath/ASME Symposium on Fluid Power and Motion Control. 2008: 357-368.

[41] BRANSON D T, WANG F C, JOHNSTON D N, et al. Piezoelectrically actuated hydraulic valve design for high bandwidth and flow performance[J]. Proceedings of the Institution of Mechanical Engineers Part I Journal of Systems \& Control Engineering, 2011, 225(3): 345-359.

[42] SELL N P, JOHNSTON N, PLUMMER A R, et al. Development of a position controlled digital hydraulic valve[C]// ASME/BATH 2015 Symposium on Fluid Power and Motion Control. American Society of
Mechanical Engineers, 2015: V001T01A008.

[43] GUAN Changbin, JIAO Zongxia, WU Shuai, et al. Active control of fluid pressure pulsation in hydraulic pipe system by bilateral-overflow of piezoelectric direct-drive slide valve[J]. Journal of Dynamic Systems Measurement \& Control, 2014, 136(3): 31025.

[44] ZHENG Huiwei, JIAO Zongxia, XU Yuanzhi, et al. Efficient active control of fluid borne pulsation in hydraulic piping systems[C]// 2016 IEEE International Conference on Aircraft Utility Systems (AUS), IEEE, 2016: 1105-1110.

[45] 季晓伟, 刘桓龙, 柯坚, 等. 基于神经网络的入流式流 体脉动主动控制[J]. 液压气动与密封, 2016，36(10): $17-21$.

JI Xiaowei, LIU Huanlong, KE Jian, et al. Active control of inflow fluid pulsation based on neural network[J]. Hydraulic Pneumatics and Sealing, 2016, 36(10): 17-21.

[46] 季晓伟, 刘桓龙, 许文学, 等. 液压管路脉动主动控制 的消振阀的设计[J]. 机械设计与制造, 2017(S1): 84-87. JI Xiaowei, LIU Huanlong, XU Wenxue, et al. Design of anti-vibration valve for active control of hydraulic piping pulsation[J]. Mechanical Design \& Manufacturing, 2017(S1): 84-87.

[47] PAN M, HILLIS A, JOHNSTON N. Active control of fluid-bome noise in hydraulic systems using in-series and by-pass structures[C]// 2014 UKACC International Conference on Control (CONTROL), IEEE, 2014 : 355-360.

[48] BRENNAN M J, ELLIOTT S J, PINNINGTON R J. A non-intrusive fluid-wave actuator and sensor pair for the active control of fluid-borne vibrations in a pipe[J]. Smart Materials \& Structures, 1996, 5(3): 281.

[49] LANG K, XIA P. Hybrid active vibration control of helicopter fuselage driven by piezoelectric stack actuators[J]. Journal of Aircraft, 2018: 1-11.

[50] MAILLARD J. Active Control of Pressure Pulsations in Piping Systems[R]. Ronneby, Sweden: Department of Applied Signal Processing, University of Karlskrona, 1998.

[51] MAILLARD J， LAGO T L，FULLER C R. Fluid wave actuator for the active control of hydraulic pulsations in piping systems[J]. Proceedings of SPIE - The International Society for Optical Engineering, 1999, 3727: 1806-1812.

[52] KIYAR M B, JOHNSON M E, FULLER C R. Experiments on the active control of multiple wave types 
in fluid filled piping systems[C]// ASME 2002 International Mechanical Engineering Congress and Exposition. American Society of Mechanical Engineers, 2002: 161-168.

[53] CHEER J, DALEY S. Broadband active control of noise and vibration in a fluid-filled pipeline using an array of non-intrusive structural actuators[C]// Inter-noise \& Noise-con Congress \& Conference, 2015.
作者简介：商夏，女，1992 年出生，博士研究生。主要研究方向为液压 系统振动与噪声控制。

E-mail: shangxia1007@163.com

周华(通信作者), 男, 1968 年出生, 博士, 教授, 博士研究生导师。主 要研究方向为水液压技术、电液控制工程。

E-mail: hzhou@sfp.zju.edu.cn

杨华勇, 男, 1961 年出生, 博士, 教授, 博士研究生导师, 中国工程院 院士。主要研究方向为电液控制元件与系统、流体动力与机电系统的 节能。

E-mail: yhy@zju.edu.cn

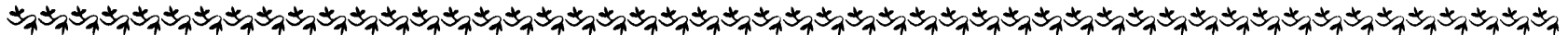

(上接第 215 页)

Engineering, 2012, 32(12): 916-921.

[14] 董康, 周吴, 杨玉, 等. 二次风量对旋流燃烧器气固流 动特性的影响 [J]. 浙江大学学报, 2014, 48(12): 2162-2171.

DONG Kang, ZHOU Hao, YANG Yu, et al. Influence of mass flow rate of secondary air on gas/solid characteristics of a swirl burner[J]. Journal of Zhejiang
University, 2014, 48(12): 2162-2171.

作者简介: 刘铭媛(通信作者), 女, 1983 年出生, 工程师。主要研究 方向为燃煤发电机组清洁高效燃烧技术和污染物控制技术，煤粉燃烧 数值模拟。

E-mail: mingyuan716@163.com

陈国庆, 男, 1982 年出生, 高级工程师。主要研究方向燃煤发电机组清 洁高效燃烧技术和污染物控制技术。

E-mail: chengqhit@163.com 